\title{
Theory of laser oscillation in resonators with photorefractive gain
}

\author{
Amnon Yariv and Sze-Keung Kwong \\ California Institute of Technology, Pasadena, California 91125
}

Received March 15, 1985; accepted June 17, 1985

\begin{abstract}
A theory for oscillation in an optical resonator with photorefractive gain was formulated. The threshold conditions for the oscillation were also obtained. The result, applicable to a whole class of new devices, is a prediction for an oscillation frequency different from that of the pump beam.
\end{abstract}

The phenomenon of two-beam coupling in photorefractive crystals has been used in the past to obtain ring-cavity oscillation in the basic configuration ${ }^{1}$ shown in Fig. 1. Such oscillation was subsequently employed to obtain self-pumped ${ }^{1-3}$ phase conjugation and may be important in gyroscopic applications.

In two recent communications, Rajbenbach and Huignard ${ }^{4}$ and Feinberg and Bacher ${ }^{5}$ reported on the observation of a frequency difference between that of the pump beam $\left(\omega_{0}\right)$ and that of the wave circulating in the ring resonator $(\omega)$.

In this Letter we apply the field theory of optical oscillation to the configuration of Fig. $1 .^{6}$ The result is a prediction for an oscillation frequency that is indeed different from that of the pump beam. The theory also provides an explanation of the observed fact that selfpumped phase-conjugate mirrors function even when the input beam is severely distorted or strongly (spatially) modulated.

Referring to Fig. 1, we take the known input (pump) beam as

$$
\mathbf{E}_{i}(\mathbf{r}, t)=1 / 2 \mathbf{E}_{i 0}(\mathbf{r}) \exp \left(i \omega_{0} t\right)+\text { c.c., }
$$

where $\mathbf{E}_{i 0}(\mathbf{r})$ contains the propagation factor as well as describing the effect of distortion and of information (spatial) modulation of the beam. The oscillating beam, which establishes itself in the ring oscillator, is taken as $\mathbf{E}(\mathbf{r}, t)$, and our immediate task is to solve for the oscillation condition and the oscillation frequency of this beam. The resonator field can be expanded in the (complete) set of the resonator mode $\mathbf{E}_{a}(\mathbf{r})^{7,8}$ :

$$
\begin{aligned}
& \mathbf{E}(\mathbf{r}, t)=\sum_{a}-\frac{1}{\sqrt{\epsilon}} p_{a}(t) \mathbf{E}_{a}(\mathbf{r}), \\
& \mathbf{H}(\mathbf{r}, t)=\sum_{a} \frac{1}{\sqrt{\mu}} \omega_{a} q_{a}(t) \mathbf{H}_{a}(\mathbf{r}),
\end{aligned}
$$

where $\mathbf{E}_{a}(\mathbf{r})$ and $\mathbf{H}_{a}(\mathbf{r})$ satisfy the resonator boundary conditions for electric and magnetic fields, respectively, $\epsilon$ and $\mu$ are electric and magnetic permittivities, respectively. In addition,

$$
\begin{aligned}
\nabla \times \mathbf{E}_{a} & =k_{a} \mathbf{H}_{a}, \\
\nabla \times \mathbf{H}_{a} & =k_{a} \mathbf{E}_{a},
\end{aligned}
$$

where $k_{a}=\omega_{a} \sqrt{\omega \epsilon}$. The quantity $p_{a}(t)$, as an example, contains the temporal information of mode $a$, including that of the frequency. In addition, the modal functions $\mathbf{E}_{a}$ and $\mathbf{H}_{a}$ are orthonormal according to

$$
\int_{V_{\mathrm{res}}} \mathbf{E}_{a} \cdot \mathbf{E}_{b} d V=\delta_{\mathrm{ab}}, \quad \int_{V_{\mathrm{res}}} \mathbf{H}_{a} \cdot \mathbf{H}_{b} \mathrm{~d} V=\delta_{\mathrm{ab}} .
$$

The insertion of Eqs. (2) into Maxwell's equation plus the use of Eqs. (3) and (4) leads to the oscillator equation $^{8}$

$$
\begin{aligned}
\ddot{p}_{a}+\frac{\omega_{a}}{Q_{a}} \dot{p}_{a} & +\omega_{a}^{2} p_{a} \\
& =\frac{1}{\sqrt{\epsilon}} \frac{\partial^{2}}{\partial t^{2}} \int_{V_{\mathrm{res}}} \mathbf{P}_{\mathrm{NL}}(\mathbf{r}, t) \cdot \mathbf{E}_{a}(\mathbf{r}) \mathrm{d} V,
\end{aligned}
$$

where $Q_{a}$ is the quality factor of the resonator for mode $a$ and $\mathbf{P}_{\mathrm{NL}}(\mathbf{r}, t)$ is the polarization in the photorefractive crystal that is due to the nonlinear interaction between the pump (input) beam $\mathbf{E}_{i}(\mathbf{r}, t)$ and the oscillator field $\mathbf{E}(\mathbf{r}, t)$. From Eq. (5) we identify $\omega_{a}$ as the resonance frequency of mode $a$ in the no-loss $\left(Q_{a} \rightarrow \infty\right)$ limit. The distributed nonlinear polarization term $\mathbf{P}_{\mathrm{NL}}(\mathbf{r}, t)$ driving the oscillation of the resonator field is that

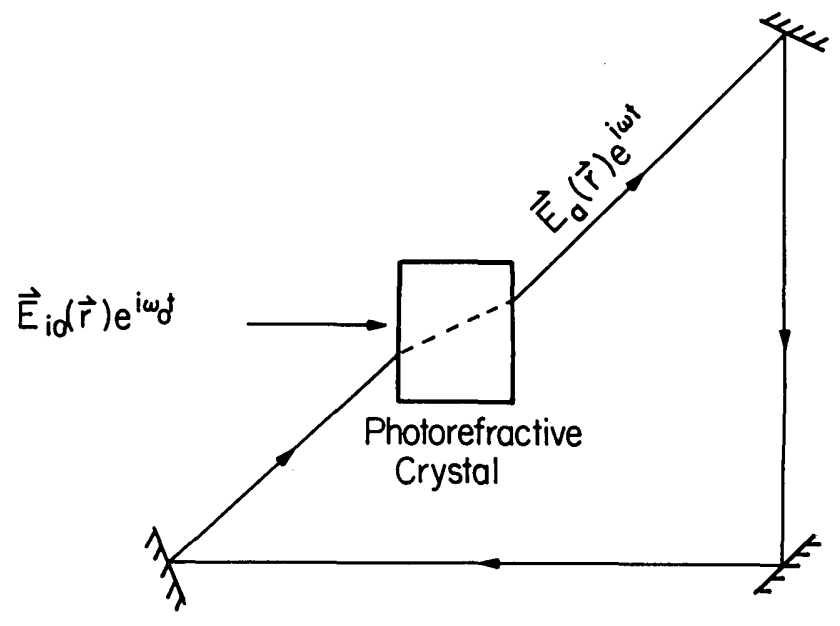

Fig. 1. Schematic diagram of a photorefractively pumped unidirectional ring resonator. 
produced by the incidence of the input field $\mathbf{E}_{i}(\mathbf{r}, t)$ on the index grating created photorefractively by the interaction of the field $\mathbf{E}_{i}(\mathbf{r}, t)$ and the ring-oscillator field $E(r, t)$.

If we assume that one mode only, say $a$, oscillates, then we may replace $\mathbf{E}(\mathbf{r}, t)$ by the $a$ th summand in Eqs. (2) and write

$$
\begin{aligned}
\mathbf{P}_{\mathrm{NL}}(\mathbf{r}, t) & =4 \epsilon_{0} \Delta n(\mathbf{r}) \mathbf{E}_{i}(\mathbf{r}, t) \\
& =-\frac{\epsilon_{0}}{\sqrt{\epsilon}} \gamma \frac{p_{a}(t)\left[\mathbf{E}_{i 0} * \mathbf{E}_{a}(\mathbf{r})\right] \mathbf{E}_{i 0}(\mathbf{r})}{\left|\mathbf{E}_{i 0}\right|^{2}+\frac{1}{\epsilon}\left|p_{a} \mathbf{E}_{a}\right|^{2}}+\text { c.c. }
\end{aligned}
$$

where $\Delta n$, the index grating formed by the interference of the input beam $\mathbf{E}_{i}(\mathbf{r}, t)$ and the oscillation field $\epsilon^{-1 / 2} p_{a}(t) \mathbf{E}_{a}(\mathbf{r})$, is given by ${ }^{9}$

$$
\Delta n(\mathbf{r}, t)=-\frac{\gamma}{2 \sqrt{\epsilon}} \frac{p_{a}(t)\left[\mathbf{E}_{i 0} * \cdot \mathbf{E}_{a}(\mathbf{r})\right] \exp \left(-i \omega_{0} t\right)}{\left|\mathbf{E}_{i 0}\right|^{2}+\frac{1}{\epsilon}\left|p_{a} \mathbf{E}_{a}\right|^{2}}
$$

The photorefractive (complex) coupling constant $\gamma$ is given ${ }^{10,11}$ by

$$
\gamma=\frac{r_{\mathrm{eff}} n_{0}{ }^{3}}{2} \frac{i E_{p}\left(E^{\prime}+i E_{d}\right)}{\left[E^{\prime}-\left(\omega-\omega_{0}\right) t_{0}\left(E_{d}+E_{\mu}\right)\right]+i\left[E_{d}+E_{p}+\left(\omega-\omega_{0}\right) t_{0} E^{\prime}\right]},
$$

$$
p_{a}(t)=p_{a 0}(t) e^{i \omega t} .
$$

In addition, we use Eq. (6) to obtain in the process

$$
\begin{aligned}
& \left\{\left[\left(\omega_{a}^{2}-\omega^{2}\right)+i \frac{\omega_{a} \omega}{Q_{a}}\right] p_{a 0}(t)\right. \\
& \left.\quad+\left(2 i \omega+\frac{\omega_{a}}{Q_{a}}\right) \dot{p}_{a 0}+\ddot{p}_{a 0}\right\} e^{i \omega t} \\
& \quad=-\frac{\epsilon_{0}}{\epsilon} \frac{\partial^{2}}{\partial t^{2}} \int_{V_{\text {crystal }}} \frac{\gamma p_{a 0}(t)\left|\mathbf{E}_{i 0} * \cdot \mathbf{E}_{a}\right|^{2}}{\left|\mathbf{E}_{i 0}\right|^{2}+\frac{1}{\epsilon}\left|p_{a 0}(t) \mathbf{E}_{a}(\mathbf{r})\right|^{2}} e^{i \tau} \mathrm{d} v .
\end{aligned}
$$

At steady state $\dot{p}_{a}$ and $\ddot{p}_{a}$ vanish, $\partial / \partial t \rightarrow i \omega$, and $p_{a 0}(t)$ $=p_{a 0}(\infty)=$ constant. The oscillation condition (11) becomes

$$
\begin{aligned}
\left(\omega_{a}^{2}-\omega^{2}\right)+i \frac{\omega_{a} \omega}{Q_{a}} & =\frac{\epsilon_{0}}{\epsilon} \omega^{2} \gamma f \\
& =i \frac{\epsilon_{0}}{\epsilon} \omega^{2} f \frac{\gamma_{0}}{1+i\left(\omega-\omega_{0}\right) \tau},
\end{aligned}
$$

where in the second equality we used the zero-external-field $\left(E^{\prime}=0\right)$ form of $\gamma$ as given by Eq. (9) and $f$ is given by where $r_{\text {eff }}$ is the relevant electro-optic coefficient, $n_{0}$ is the refractive index of the crystal, $t_{0}$ is the characteristic time, $E^{\prime}$ is the externally applied electric dc field, and $E_{\mu}, E_{d}$, and $E_{p}$ are internal electric fields characteristic of drift, diffusion, and maximum space charge, respectively. The parameters $r_{\text {eff }}, t_{0}, E_{\mu}, E_{d}$, and $E_{p}$ can be calculated from given crystal parameters and crystal orientation with respect to the various interacting beams. $\omega$ and $\omega_{0}$, we recall, are the frequency of the oscillating mode $a$ (as yet unknown) and that of the pump beam, respectively. If there is no applied electric field, $E^{\prime}=0$, and

$$
\gamma=\frac{i \gamma_{0}}{1+i\left(\omega-\omega_{0}\right) \tau}
$$

where

$$
\gamma_{0}=\frac{r_{\mathrm{eff}} n_{0}^{3}}{2} \frac{E_{p} E_{d}}{\left(E_{p}+E_{d}\right)}
$$

and

$$
\tau=t_{0}\left(\frac{E_{d}+E_{\mu}}{E_{d}+E_{p}}\right) .
$$

We note that the sole time dependence of $\mathbf{P}_{\mathrm{NL}}(\mathbf{r}, t)$ is that of mode $a$, i.e., of the term $p_{a}(t)$. The time dependence of the input mode $\mathbf{E}_{i}(\mathbf{r}, t)$ has disappeared since $\mathbf{E}_{i}$ appears in Eq. (6) multiplied by its complex conjugate. An equivalent way to explain this fact is that the index grating produced by the interference of $\mathbf{E}_{i}$ and $\mathbf{E}_{a}$ (the cavity field) is moving since $\omega \neq \omega_{0}$, and this velocity is just the right one to Doppler shift the incident frequency $\omega_{0}$ to $\omega$.

Returning to the oscillation equation (5), we take $p_{a}(t)$ as the product of a slowly varying amplitude $p_{a 0}(t)$ and an optical oscillation term $\exp (i \omega t)$ :

$$
f=\int_{V_{\text {crystal }}} \frac{\left|\mathbf{E}_{i 0} *(\mathbf{r}) \cdot \mathbf{E}_{a}(\mathbf{r})\right|^{2}}{\left|\mathbf{E}_{i 0}(\mathbf{r})\right|^{2}+\frac{1}{\epsilon}\left|p_{a 0}(\infty) \mathbf{E}_{a}(\mathbf{r})\right|^{2}} \mathrm{~d} V
$$

so that it is dimensionless and real.

The left-hand side of Eq. (12) is a complex number that depends only on passive resonator parameters and the (yet unknown) oscillation frequency $\omega$. According to Eq. (6), the phase of the right-hand side of Eq. (6) depends on $\left(\omega-\omega_{0}\right)$. The frequency $\omega$ will thus adjust itself relative to $\omega_{0}$ to satisfy Eq. (12). Using Eq. (6) and separating the real and imaginary parts of Eq. (12) lead to

$$
\omega_{a}^{2}-\omega^{2}=\frac{\epsilon_{0} f \gamma_{0} \omega^{2}\left(\omega-\omega_{0}\right) \tau}{\epsilon\left[1+\left(\omega-\omega_{0}\right)^{2} \tau^{2}\right]}
$$

and

$$
\frac{\omega_{a} \omega}{Q_{a}}=\frac{\epsilon_{0} f \gamma_{0} \omega^{2}}{\epsilon\left[1+\left(\omega-\omega_{0}\right)^{2} \tau^{2}\right]} .
$$

Since $\omega \approx \omega_{a} \approx \omega_{0}$, Eq. (14) can be approximated to a high degree of accuracy by

$$
\omega_{a}-\omega=\frac{\epsilon_{0} f \gamma_{0} \omega_{0}\left(\omega-\omega_{0}\right) \tau}{2 \epsilon\left[1+\left(\omega-\omega_{0}\right)^{2} \tau^{2}\right]} .
$$

In the limit $t_{a} \ll \tau$, where $t_{a}=Q_{a} / \omega_{a}$ is the decay time constant of the photon density in the $a$ th mode (with no photorefractive interaction), we can solve Eq. (16) for $\omega$ and, using Eq. (15), obtain

$$
\left(\omega-\omega_{0}\right) \cong 2 \frac{t_{a}}{\tau}\left(\omega_{a}-\omega_{0}\right) .
$$

This is our main result. It predicts a shift between the frequency of oscillation $(\omega)$ and that of the pumping 
beam $\left(\omega_{0}\right)$. The dependence of this shift on the parameters indicated in the equation was verified experimentally both in a ring resonator pumped photorefractively ${ }^{12}$ and in a resonator pumped by two photorefractive phase-conjugate mirrors. ${ }^{13}$ These results will be described in a future publication.

Let us return next to the threshold condition (15). The parameter $f$ is given by Eq. (13) and can be written as

$$
\begin{aligned}
f & \equiv \int \frac{\left|\mathbf{E}_{i 0} *(\mathbf{r}) \cdot \mathbf{E}_{a}(\mathbf{r})\right|^{2}}{\left|\mathbf{E}_{i 0}(\mathbf{r})\right|^{2}+\left|\mathbf{E}_{\mathrm{osc}}(\mathbf{r})\right|^{2}} \mathrm{~d} V \\
& \cong \frac{1}{1+\frac{\left\langle\left|\mathbf{E}_{\mathrm{osc}}\right|^{2}\right\rangle}{\left\langle\left|\mathbf{E}_{i 0}\right|^{2}\right\rangle}} \int_{V_{\mathrm{c}}} \frac{\left|\mathbf{E}_{i 0} 0^{*}(\mathbf{r}) \cdot \mathbf{E}_{a}(\mathbf{r})\right|^{2}}{\left|\mathbf{E}_{i 0}\right|^{2}} \mathrm{~d} V,
\end{aligned}
$$

where we used the first of Eqs. (2) to write the oscillating electric field of the $a$ th mode as

$$
\mathbf{E}_{\mathrm{osc}}(\mathbf{r})=-\frac{1}{\sqrt{\epsilon}} p_{a 0}(\infty) \mathbf{E}_{a}(\mathbf{r})
$$

and \langle\rangle to denote spatial averaging over the crystal volume. We can now rewrite $f$ as

$$
f=\frac{f_{0}}{1+\frac{\left\langle\left|\mathbf{E}_{\text {osd }}\right|^{2}\right\rangle}{\left\langle\left|\mathbf{E}_{i 0}\right|^{2}\right\rangle}},
$$

where

$$
f_{0} \equiv \int \frac{\left|\mathbf{E}_{i 0} *(\mathbf{r}) \cdot \mathbf{E}_{a}(\mathbf{r})\right|^{2}}{\left|\mathbf{E}_{i 0}\right|^{2}} \mathrm{~d} V
$$

and Eq. (15) becomes

$$
\frac{1}{\omega_{a} t_{a}}=\frac{n^{2} \gamma_{0} f_{0}}{1+4\left(\omega_{0}-\omega_{a}\right)^{2} t_{a}^{2}}\left(\frac{1}{1+\frac{\left\langle\left|\mathbf{E}_{\mathrm{osc}}\right|^{2}\right\rangle}{\left\langle\left|\mathbf{E}_{i 0}\right|^{2}\right\rangle}}\right),
$$

where $t_{a}=Q_{a} / \omega_{a}$ is the resonator- (ath-) mode decay time constant. At the transient start of oscillation, the right-hand side of Eq. (22) with $\mid \mathbf{E}_{\text {osd }}=0$ must be larger than (or equal to) the left-hand side. Once oscillation starts, the term $\left|\mathbf{E}_{\text {osc }}\right|^{2}$ will grow until both sides are equal. The start-oscillation condition is thus

$$
\gamma_{0} \geq \frac{1+4\left(\omega_{0}-\omega_{a}\right)^{2} t_{a}{ }^{2}}{n^{2} f_{0} \omega_{a} t_{a}}
$$

and does not depend on the pumping intensity $\left|\mathbf{E}_{i 0}\right|^{2}$.

Equation (22) can be solved for the oscillating-field intensity inside the resonator:

$$
\left\langle\left|\mathbf{E}_{\mathrm{osc}}\right|^{2}\right\rangle=\left\langle\left|\mathbf{E}_{i 0}\right|^{2}\right\rangle\left[\frac{n^{2} \gamma_{0} f_{0} \omega_{a} t_{a}}{1+4\left(\omega_{0}-\omega_{a}\right)^{2} t_{a}^{2}}-1\right],
$$

which is reminiscent of the expression for the power output of homogeneously broadened lasers. ${ }^{14}$

We have neglected in our analysis the change in intensity of both the pump and the resonator beams in the crystal owing to the mutual power exchange. This neglect is well justified near threshold, and even a 20-30\% power exchange per pass will not invalidate the basic conclusions of the above analysis. Another important issue is the relationship of distortion (or intentional spatial modulation) of the pumping beam $\mathbf{E}_{i 0}$ to the oscillation. It follows from Eq. (21) that the main effect is to reduce the projection of $\mathbf{E}_{i 0}$ on $\mathbf{E}_{a}$, leading to a smaller $f_{0}$ and thus, according to expression (23), to a higher threshold. The shape $\mathbf{E}_{a}(\mathbf{r})$ of the oscillating field is not affected.

In summary: We have formulated an oscillator theory for photorefractively pumped resonators. Some basic features of the analysis are consistent with observations made to date. Other aspects await confirmation. The main result, which is applicable to a whole class of new devices, is a prediction of and an expression for a frequency offset between the input frequency and that of the oscillation field in optical resonators with photorefractively induced gain.

This research was supported by the U.S. Air Force Office of Scientific Research and the U.S. Army Research Office, Durham, North Carolina.

\section{References}

1. J. O. White, M. Cronin-Golomb, B. Fischer, and A. Yariv, Appl. Phys. Lett. 40, 450 (1982).

2. M. Cronin-Golomb, B. Fischer, J. O. White, and A. Yariv, Appl. Phys. Lett. 41, 689 (1982).

3. J. Feinberg, Opt. Lett. 7, 486 (1982).

4. H. Rajbenbach and J. P. Huignard, Opt. Lett. 10, 137 (1985).

5. J. Feinberg and G. D. Bacher, Opt. Lett. 9, 420 (1984).

6. For the sake of specificity we consider the case of a ring resonator. The analysis is more general and applies to any resonator with photorefractive gain.

7. J. C. Slater, Microwave Electronics (Van Nostrand, Princeton, N.J., 1950).

8. A. Yariv, Quantum Electronics, 2nd ed. (Wiley, New York, 1975).

9. B. Fischer, M. Cronin-Golomb, J. O. White, and A. Yariv, Opt. Lett. 6, 519 (1981).

10. M. Cronin-Golomb, Ph.D. dissertation (California Institute of Technology, Pasadena, California, 1983; unpublished).

11. J. P. Huignard and A. Marrackchi, Opt. Commun. 38, 249 (1981).

12. S. K. Kwong, A. Yariv, M. Cronin-Golomb, and I. Ury, Appl. Phys. Lett. (to be published).

13. M. Cronin-Golomb, B. Fischer, S. K. Kwong, J. O. White, and A. Yariv, Opt. Lett. 10, 353 (1985).

14. A. Yariv, Optical Electronics, 3rd ed. (Holt, Rinehart and Winston, New York, 1985), p. 159, Eq. (6.4-13). 